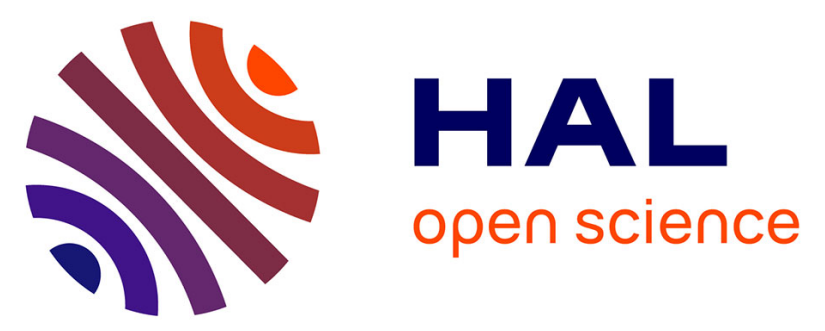

\title{
Méthode itérative de calcul des concentrations en éléments donneurs et accepteurs d'un semiconducteur faiblement dopé: application au cas de GaAs de type n
}

Didier Lemoine, C. Pelletier, S. Rolland, R. Granger

\section{- To cite this version:}

Didier Lemoine, C. Pelletier, S. Rolland, R. Granger. Méthode itérative de calcul des concentrations en éléments donneurs et accepteurs d'un semiconducteur faiblement dopé: application au cas de GaAs de type n. Revue de Physique Appliquée, 1977, 12 (5), pp.877-883. 10.1051/rphysap:01977001205087700 . jpa-00244254

HAL Id: jpa-00244254

https://hal.science/jpa-00244254

Submitted on 1 Jan 1977

HAL is a multi-disciplinary open access archive for the deposit and dissemination of scientific research documents, whether they are published or not. The documents may come from teaching and research institutions in France or abroad, or from public or private research centers.
L'archive ouverte pluridisciplinaire $\mathbf{H A L}$, est destinée au dépôt et à la diffusion de documents scientifiques de niveau recherche, publiés ou non, émanant des établissements d'enseignement et de recherche français ou étrangers, des laboratoires publics ou privés. 
Classification

Physics Abstracts

$8.220-8.224-8.272$

\title{
MÉTHODE ITÉRATIVE DE CALCUL DES CONCENTRATIONS EN ÉLÉMENTS DONNEURS ET ACCEPTEURS D'UN SEMICONDUCTEUR FAIBLEMENT DOPÉ : APPLICATION AU CAS DE GaAs DE TYPE n
}

\author{
D. LEMOINE, C. PELletiER, S. ROLLAND et R. GRANGER
}

Institut National des Sciences Appliquées, Département de Génie Physique, 35031 Rennes Cedex, France

(Reçu le 13 janvier 1977, accepté le 1er février 1977)

\begin{abstract}
Résumé. - Lors de la recherche des concentrations en impuretés dans un semiconducteur, il a toujours été fait abstraction jusqu'à présent de la valeur réelle du facteur de dispersion $r_{\text {H }}$ qui a été prise constante et égale à 1 . Or, cette valeur peut dans certains cas atteindre et même dépasser 1,5. Une méthode numérique itérative est développée ici dans le cas de $\mathrm{GaAs}$ de type $\mathrm{n}$, permettant de calculer à la fois la valeur du facteur de dispersion $r_{H}$ en fonction de la température et les concentrations en impuretés de l'échantillon considéré.
\end{abstract}

\begin{abstract}
Up to now, the methods used to obtain the concentrations of impurities in a semiconductor disregard the actual value of the dispersion factor $r_{H}$ which has been taken constant and equal to 1 . However, this dispersion factor is not a constant and its values can reach and even exceed 1.5 in some cases. A numerical iterative method is proposed here for $\mathrm{n}-\mathrm{GaAs}$ that enable both the value of the dispersion factor $r_{\mathrm{H}}$ as a function of temperature and impurity concentrations of the sample to be calculated.
\end{abstract}

1. Introduction. - Les propriétés d'un matériau semi-conducteur dépendent de sa concentration en impuretés qu'il est donc important de connaître avec précision. Jusqu'alors, cette détermination a généralement été effectuée à partir des mesures électriques de résistivité $\rho$ et de mobilité de Hall $\mu_{\mathrm{H}}$ et selon deux méthodes :

- La première consiste en l'étude de la variation de la concentration électronique en fonction de la température dans le domaine d'ionisation des centres d'impuretés [1]. Considérant le cas des matériaux de type $\mathrm{n}$, qui sont étudiés ici, la densité en électrons libres est liée à la valeur de la concentration en centres donneurs $N_{\mathrm{D}}$, à celle en centres accepteurs $N_{\mathrm{A}}$ et à l'énergie d'activation thermique $\varepsilon_{\mathrm{D}}$ des électrons du niveau d'impuretés donneur vers la bande de conduction. La détermination de $N_{\mathrm{D}}, N_{\mathrm{A}}$ et $\varepsilon_{\mathrm{D}}$ s'effectue alors à partir de la variation de la concentration en électrons de la bande de conduction en fonction de la température $T$ en utilisant une technique de moindres carrés ; ces concentrations électroniques sont déduites directement des mesures expérimentales de résistivité et de mobilité de Hall.

- La seconde méthode repose sur l'analyse de la mobilité des porteurs libres en fonction de la température [2] : celle-ci dépend des concentrations $N_{\mathrm{D}}$ et $N_{\mathrm{A}}$ de manière significative quand les mécanismes de diffusion sur les impuretés à l'état neutre ou ionisé prennent une part appréciable à la limitation de mobilité des porteurs libres. Dans une première approche, la relation de Brooks-Herring [3] qui relie le temps de relaxation correspondant à la dispersion par les impuretés ionisées aux paramètres $N_{\mathrm{D}}$ et $N_{\mathrm{A}}$, fut le plus souvent utilisée seule pour estimer les densités en éléments d'impuretés : le domaine de température sur lequel l'analyse est réalisée est alors choisi en supposant que l'effet des autres processus de dispersion peut être négligé [4].

Le point faible de ces deux types d'investigation est de confondre dans tous les cas, mobilité de conduction $\mu$ et mobilité de Hall $\mu_{\mathrm{H}}$ ce qui équivaut à prendre 1 pour valeur du facteur de diffusion $r_{\mathrm{H}}$. Or, la valeur de ce terme $r_{\mathrm{H}}$ dépend essentiellement du mode de dispersion des électrons et de ce fait, varie avec la température. Ainsi, dans le cas de GaAs de type n, si la simplification consistant à considérer $r_{\mathrm{H}}$ égal à l'unité peut encore se justifier lorsque la dispersion des électrons est due aux modes optiques polaires (l'erreur est alors estimée de l'ordre de $20 \%[5,8]$, il en est autrement lorsque le processus de diffusion dépend fortement de l'énergie d'un porteur libre comme c'est le cas pour la dispersion par les impuretés ionisées [6]. Le facteur de diffusion $r_{\mathrm{H}}$ peut alors atteindre et même dépasser la valeur 1,5 , sa valeur dépendant du dopage et de la température; prendre $r_{\mathrm{H}}=1$ revient ainsi à faire une erreur systématique importante. 
La nouvelle méthode d'investigation de type itératif qui est proposée ici permet de calculer le facteur de diffusion $r_{\mathrm{H}}$ et tient compte effectivement de sa valeur dans la détermination de $N_{\mathrm{D}}, N_{\mathrm{A}}$ et $\varepsilon_{\mathrm{D}}$. Elle sera appliquée à titre d'illustration au cas de GaAs de type $\mathrm{n}$.

2. Principe de la méthode. - Les résultats expérimentaux nécessaires pour conduire cette analyse sont la résistivité $\rho(T)$ et la mobilité de Hall $\mu_{\mathrm{H}}(T)$, la valeur de cette dernière étant obtenue pour des valeurs de l'induction magnétique faibles. On déduit alors la concentration électronique de Hall $n_{\mathrm{H}}(T)$ par :

$$
n_{\mathrm{H}}(T)=\frac{1}{e \cdot \rho(T) \cdot \mu_{\mathrm{H}}(T)} .
$$

Les grandeurs $\mu_{\mathrm{H}}(T)$ et $n_{\mathrm{H}}(T)$ sont reliées respectivement à la mobilité de conduction $\mu(T)$ et à la concentration réelle d'électrons libres $n(T)$ par les relations :

$$
\mu_{\mathrm{H}}(T)=r_{\mathrm{H}}(T) \cdot \mu(T)
$$

et

$$
n_{\mathrm{H}}(T)=\frac{n(T)}{r_{\mathrm{H}}(T)} .
$$

Dans le cas où la dispersion des porteurs est due à un processus élastique caractérisé par un temps de relaxation $\tau(\varepsilon)$, la mobilité de Hall $\mu_{\mathrm{H}}$ pour un matériau non dégénéré s'exprime, dans la limite des faibles inductions magnétiques, par la relation [7]:

$$
\mu_{\mathrm{H}}=\frac{e}{m^{*}} \cdot \frac{\left\langle\tau^{2}\right\rangle}{\langle\tau\rangle}=\mu \frac{\left\langle\tau^{2}\right\rangle}{\langle\tau\rangle^{2}}=\mu . r_{\mathrm{H}}
$$

où $m^{*}$ est la masse effective de l'électron,

$\langle\tau\rangle$ est une moyenne du temps de relaxation, avec $\left\langle\tau^{p}\right\rangle$ exprimée par :

$$
\left.<\tau^{p}\right\rangle=\frac{4}{3 \pi^{1 / 2}} \int_{0}^{\infty} \tau^{(p)}(x) \cdot x^{3 / 2} \exp (-x) \cdot \mathrm{d} x
$$

où $x$ est l'énergie d'un électron en unités $k_{\mathrm{B}} T$

$$
\left(x=\varepsilon / k_{\mathrm{B}} T\right) .
$$

Le principe de cette méthode d'analyse proposée repose entièrement sur la possibilité de décrire les mécanismes de dispersion des électrons par l'intermédiaire d'un temps de relaxation $\tau(\varepsilon)$, ce qui impose une limitation du domaine de température analysé. Dans ces conditions, le calcul du facteur de dispersion $r_{\mathrm{H}}(T)$ revient à calculer l'expression $\left\langle\tau^{2}\right\rangle /\langle\tau\rangle^{2}$ ainsi qu'elle apparaît dans la relation (4).

2.1 DesCRiption SChÉMATIQUe DE LA MÉTHODE. Pour GaAs de type n, les centres donneurs sont pratiquement tous ionisés à la température ambiante ce qui permet d'estimer $N_{\mathrm{D}}-N_{\mathrm{A}}$ à partir de la mesure de la concentration électronique $n_{\mathrm{H}}(300 \mathrm{~K})$ compte tenu du facteur de dispersion, ce qui conduit à :

$$
N_{\mathrm{D}}-N_{\mathrm{A}} \approx r_{\mathrm{H}}(300 \mathrm{~K}) \cdot n_{\mathrm{H}}(300 \mathrm{~K}) \text {. }
$$

Ce terme $r_{\mathrm{H}}(300 \mathrm{~K})$, non connu au départ, apparaît comme le seul paramètre ajustable de cette méthode : en effet, à partir d'une valeur choisie a priori pour $r_{\mathrm{H}}(300 \mathrm{~K})$, (de l'ordre de 1,1 en se basant sur les travaux de Kravchenko [8]), il est possible de se fixer une première évaluation de $N_{\mathrm{D}}-N_{\mathrm{A}}$ en utilisant la relation (6).

Pour un échantillon, l'analyse est limitée à un domaine de température compris entre $T_{1}$ et $T_{\mathrm{M}}$, et nous appellerons cycle de calcul numéro $p$ l'ensemble des opérations permettant d'obtenir les valeurs de $\mu$, $\mu_{\mathrm{H}}$ et $r_{\mathrm{H}}$ à partir d'une liste de concentrations électroniques $n^{(p)}\left(T_{\mathbf{i}}\right)$ en fonction de la température $T_{\mathbf{i}}$ (avec $T_{1}<T_{\mathrm{i}}<T_{\mathrm{M}}$ ) (cf. Fig. 1). Chacune des variables intervenant dans un cycle est affectée d'une part du numéro d'ordre $p$ de celui-ci et d'autre part, de l'abréviation "exp " lorsqu'il s'agit d'une valeur obtenue expérimentalement ou "calc» lorsqu'elle résulte d'un calcul numérique. Les valeurs intermédiaires de calcul sont notées généralement sans abréviation.

Un cycle numéro $p$ débute par le calcul des concentrations électroniques $n^{(p)}\left(T_{\mathrm{i}}\right)$ à partir des valeurs expérimentales $n_{\mathrm{H}}^{\exp }\left(T_{\mathrm{i}}\right)$ sous la forme :

$$
n^{(p)}\left(T_{\mathrm{i}}\right)=n_{\mathrm{H}}^{\exp }\left(T_{\mathrm{i}}\right) \cdot r_{\mathrm{H}}^{(p-1)}\left(T_{\mathrm{i}}\right) .
$$

$\mathrm{Au}$ début du premier cycle $(p=1)$ les valeurs de $r_{\mathrm{H}}^{(p=0)}\left(T_{\mathrm{i}}\right)$ sont prises égales à 1 ; à la fin du cycle numéro $p$ la valeur de $r_{\mathrm{H}}^{(p)}(T)$ qui sera utilisée dans le cycle $(p+1)$ est :

$$
r_{\mathrm{H}}^{(p)}\left(T_{\mathrm{i}}\right)=\frac{r_{\mathrm{H}}^{(p-1)}\left(T_{\mathrm{i}}\right)+r_{\mathrm{H}}^{(p) \text { calc }}\left(T_{\mathrm{i}}\right)}{2} .
$$

Comme cela apparaît sur la figure 1, le déroulement d'un cycle se décompose essentiellement en deux parties :

- la première partie consiste en l'évaluation des concentrations $N_{\mathrm{D}}$ et $N_{\mathrm{A}}$ et de l'énergie d'activation thermique $\varepsilon_{\mathbf{D}}$ à partir de $N_{\mathbf{D}}-N_{\mathbf{A}}$ et des densités électroniques $n^{(p)}\left(T_{\mathfrak{i}}\right)$ en utilisant l'équation de neutralité (cf. $\S$ suivant) et une méthode de moindres carrés ;

- dans la seconde partie on calcule à partir des valeurs de $N_{\mathrm{D}}, N_{\mathrm{A}}$ et $n^{(p) \text { calc }}\left(T_{\mathrm{i}}\right)$ obtenues, les temps de relaxation concernant les mécanismes de dispersion des électrons ; on évalue ensuite les mobilités $\mu^{(p) \text { calc }}\left(T_{\mathrm{i}}\right), \mu_{\mathrm{H}}^{(p) \text { calc }}\left(T_{\mathrm{i}}\right)$ et le facteur de dispersion $r_{\mathrm{H}}^{(p) c a l c}\left(T_{\mathrm{i}}\right)$ à l'aide des relations (4). Si l'écart relatif entre les valeurs de $\mu_{\mathrm{H}}^{(p-1) \text { calc }}\left(T_{\mathrm{i}}\right)$ et $\mu_{\mathrm{H}}^{(p) \text { calc }}\left(T_{\mathrm{i}}\right)$ est constaté trop important (cf. test défini par la relation (18), un nouveau cycle de calcul est effectué en partant de nouvelles valeurs de $r_{\mathrm{H}}\left(T_{\mathrm{i}}\right)$ définies par la relation (8). Dans le cas contraire, on compare la liste des valeurs de $\mu_{\mathrm{H}}^{(p) c a l c}\left(T_{\mathrm{i}}\right)$ avec celles des valeurs expérimentales $\mu_{\mathrm{H}}^{\exp }\left(T_{\mathrm{i}}\right)$. Si l'écart entre les valeurs expérimentales et calculées de la mobilité de Hall est trop important (cf. test défini par la relation (19) la valeur de $r_{\mathrm{H}}$ $(300 \mathrm{~K})$ est modifiée en conséquence et les cycles de 


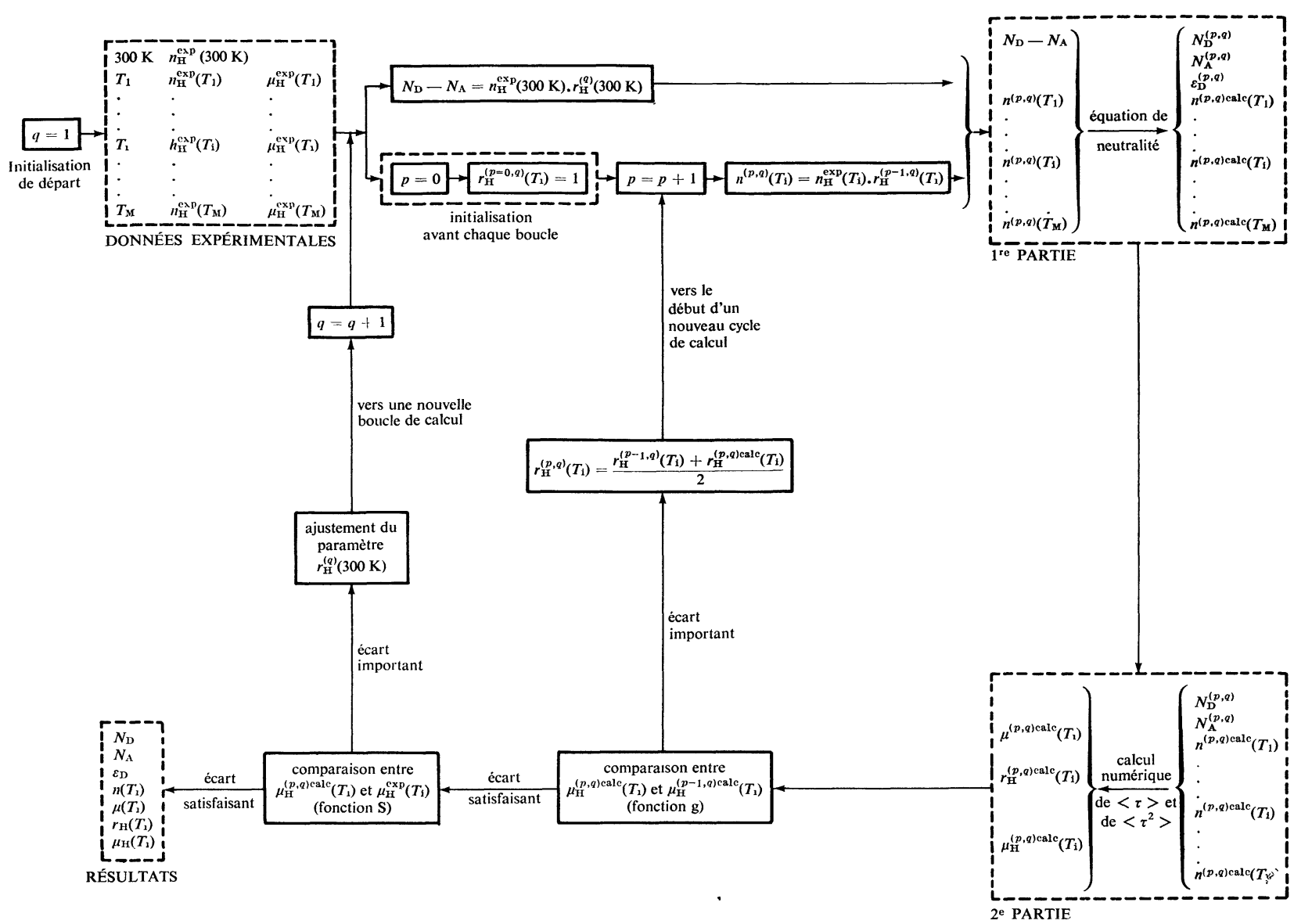

Fig. 1. - Schéma de principe de l'analyse numérique.

[Diagram of the numerical analysis.]

calcul précédents sont repris à partir de cette nouvelle valeur du facteur de diffusion à température ambiante. Un ensemble de cycles correspondant à une même valeur de $r_{\mathbf{H}}(300 \mathrm{~K})$ est appelé une boucle. On réalise ainsi plusieurs boucles de manière à avoir le meilleur accord entre les valeurs de $\mu_{\mathrm{H}}\left(T_{\mathrm{i}}\right)$ calculées et les valeurs expérimentales par ajustement de $r_{\mathbf{H}}(300 \mathrm{~K})$. L'ordre d'une boucle est défini par $q$ : en particulier, chaque variable intervenant à l'intérieur d'un cycle sera affectée des deux indices $p$ et $q$, précisant son appartenance au $p$-ième cycle de la q-ième boucle (cf. Fig. 1).

2.2 DÉTAIL D'UN CYCLE. - 1 $1^{\text {re }}$ partie: recherche des valeurs de $N_{\mathrm{D}}, N_{\mathrm{A}}$ et $\varepsilon_{\mathrm{D}}$. A une température $T$ où le régime de conduction est extrinsèque, les électrons apportés par les centres donneurs se trouvent soit localisés sur les niveaux fondamental et excités de ces centres, soit thermiquement activés dans la bande de conduction en densité $n(T)$.

Pour un semi-conducteur non dégénéré de type $\mathrm{n}$ ayant une bande de conduction parabolique à symétrie sphérique, l'équation de neutralité s'exprime par (1)

$$
\frac{n(T) \cdot\left[N_{\mathrm{A}}+n(T)\right]}{\left[N_{\mathrm{D}}-N_{\mathrm{A}}-n(T)\right]}=\frac{N_{\mathrm{C}}}{\sum_{l} \frac{1}{\beta_{l}} \cdot \exp \left[\frac{\varepsilon_{l}-\varepsilon_{\mathrm{F}}}{k_{\mathrm{B}} T}\right]}
$$

avec

$$
N_{\mathrm{C}}=2\left[\frac{2 \pi m^{*} k_{\mathrm{B}} T}{h^{2}}\right]^{3 / 2}
$$

et $\beta_{l}$, facteur de dégénérescence du niveau $l$.

$\varepsilon_{l}$, énergie du niveau $l$.

$\varepsilon_{\mathrm{F}}$, énergie de Fermi.

En analysant le nombre de porteurs de charge participant à la conduction dans GaAs de type n, Eddolls [1] a montré que lorsque l'énergie d'activation thermique $\varepsilon_{\mathrm{D}}$ est inférieure à $4 \mathrm{meV}$, l'ensemble des états excités des atomes donneurs forme un quasi continuum prolongeant l'extrémum de la bande de conduction. Dans ce cas, l'expression (9) se simplifie et donne :

$$
\frac{n(T) \cdot\left[N_{\mathrm{A}}+n(T)\right]}{\left[N_{\mathrm{D}}-N_{\mathrm{A}}-n(T)\right]}=N_{\mathrm{C}} \cdot \beta \cdot \exp \left[-\frac{\varepsilon_{\mathrm{D}}}{k_{\mathrm{B}} T}\right]
$$

où $\beta$ est très proche de $1 / 2$.

L'équation (10) est utilisée pour évaluer $N_{\mathrm{D}}, N_{\mathrm{A}}$ et $\varepsilon_{\mathrm{D}}$ par une méthode de moindres carrés.

$2^{\mathrm{e}}$ partie : recherche des mobilités $\mu$ et $\mu_{\mathrm{H}}$ et $d u$ facteur de Hall $r_{\mathrm{H}^{\bullet}}$ - Cette seconde partie d'un cycle de calcul est basée sur l'analyse des mécanismes de dispersion des électrons libres sur un domaine de tem- 
pérature où la dispersion par les impuretés ionisées est importante. Dans le cas de GaAs de type n, il est maintenant bien connu [2], [6] que ce sont les diffusions par les phonons acoustiques via le potentiel de déformation et le couplage piézoélectrique ainsi que la dispersion par les impuretés neutres qui interviennent simultanément avec la diffusion par les impuretés ionisées pour limiter la mobilité électronique aux basses températures. Tous les mécanismes de dispersion cités ci-dessus correspondent à des processus quasi élastiques et peuvent être décrits séparément par un temps de relaxation. Le temps de relaxation traduisant l'effet de tous les mécanismes est alors donné par [2] :

$$
\frac{1}{\tau(\varepsilon)}=\sum_{i} \frac{1}{\tau_{i}(\varepsilon)}
$$

$\tau_{i}(\varepsilon)$ étant le temps de relaxation correspondant à chacun des processus individuels et qui sont :

- potentiel de déformation (phonons acoustiques) [9]

$$
\frac{1}{\tau_{\mathrm{def}}(\varepsilon)}=\frac{2^{1 / 2}}{\pi \hbar^{4}} \frac{m^{* 3 / 2} \cdot E_{1}^{2}}{C_{l}} k_{\mathrm{B}} T \cdot \varepsilon^{1 / 2}
$$

où $E_{1}$ est le potentiel de déformation et $C_{l}$, la constante d'élasticité longitudinale ;

- couplage piézoélectrique (phonons acoutisques) [10]

$\frac{1}{\tau_{\text {piézo }}(\varepsilon)}=\frac{e^{2} \sqrt{2}}{\pi \hbar^{2} 35} m^{* 1 / 2} \cdot h_{14}^{2}\left(\frac{3}{C_{l}}+\frac{4}{C_{\mathrm{t}}}\right) k_{\mathrm{B}} T \cdot \varepsilon^{1 / 2}$

où $h_{14}$ est la constante piézoélectrique et $C_{\mathrm{t}}$ la constante d'élasticité transversale ;

- impuretés neutres [11]

$$
\frac{1}{\tau_{\mathrm{N}}(\varepsilon)}=\frac{20}{8 \pi^{3} m^{* 2} e^{2}}
$$

où $N_{\mathrm{n}}$ est la densité d'impuretés neutres et $\chi$ la constante diélectrique statique ;

- impuretés ionisées (relation de Brooks-Herring) [3]

$$
\frac{1}{\tau_{\mathrm{I}}(\varepsilon)}=\frac{\pi e^{4}\left(2 N_{\mathrm{A}}+n\right)}{\chi^{2}\left(2 m^{*}\right)^{1 / 2}}\left[\ln (1+b)-\frac{b}{1+b}\right] \cdot \varepsilon^{-3 / 2}
$$

avec

$$
b=\frac{2 m^{*} \chi k_{\mathrm{B}} T}{\pi \hbar^{2} e^{2} n^{*}}
$$

et

$$
n^{*}=n+\left[\left(n+N_{\mathrm{A}}\right) \frac{N_{\mathrm{D}}-N_{\mathrm{A}}-n}{N_{\mathrm{D}}}\right] .
$$

Une correction tenant compte de l'interaction électronélectron [12] nécessaire lorsque la concentration électronique est supérieure à $10^{15} \mathrm{~cm}^{-3}$ est introduite $\operatorname{sur} \tau_{\mathbf{I}}(\varepsilon)$ sous la forme [6]

$\tau_{\mathrm{I}}^{\prime}=\tau_{\mathrm{I}}\left[1-\frac{1}{2 !} \frac{n}{2 N_{\mathrm{A}}+n}+\frac{1}{3 !}\left(\frac{n}{2 N_{\mathrm{A}}+n}\right)^{2}\right]$

$\tau_{\mathrm{I}}^{\prime}$ représentant alors le temps de relaxation de la dispersion sur les impuretés ionisées incluant l'effet des collisions interélectroniques.

Les valeurs obtenues en fin de première partie de cycle pour $N_{\mathrm{D}}, N_{\mathrm{A}}$ et $n^{(p, q) \text { calc }}\left(T_{\mathrm{i}}\right)$ permettent le calcul du temps de relaxation global $\tau(\varepsilon)$ selon la relation (11), puis le calcul de la moyenne de $\tau(\varepsilon)$ et de $\tau^{2}(\varepsilon)$ d'après la relation (5). Ces valeurs conduisent à la détermination de la mobilité de conduction $\mu^{(p, q) \text { calc }}\left(T_{\mathbf{i}}\right)$ de la mobilité de Hall $\mu_{\mathrm{H}}^{(p, q) \text { calc }}\left(T_{\mathbf{i}}\right)$ et du facteur de dispersion $r_{\mathrm{H}}^{(p, q) \text { calc }}\left(T_{\mathrm{i}}\right)$ à l'aide des relations (2) et (4). Comme nous l'avons déjà précisé, la connaissance de ces valeurs oriente la suite de l'analyse soit vers le commencement d'un nouveau cycle, soit vers le commencement d'une nouvelle boucle de cycles ou vers l'arrêt du calcul et la sortie des résultats finals.

3. Remarques. - Le domaine de température où l'analyse numérique est effectuée, doit être choisi et limité en fonction des deux critères suivants :

- La conduction par impuretés (de type hopping ou métallique) doit toujours y être suffisamment faible pour pouvoir être négligée par rapport à la conduction due aux électrons libres. Ceci conduit à limiter inférieurement le domaine de température considéré.

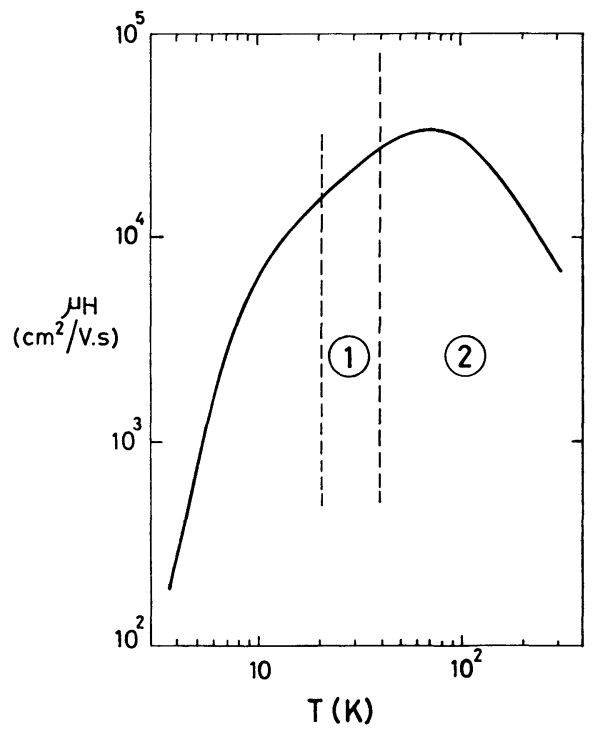

FIG. 2. - Variation de la mobilité de Hall $\mu_{\mathrm{H}}$ en fonction de la température $T$ pour un échantillon de n-GaAs. 1) Zone de température où la dispersion est caractérisée par un temps de relaxation (domaine de l'analyse). 2) Zone de température où la dispersion n'est pas caractérisée par un temps de relaxation (phonons optiques essentiellement).

[Temperature variation of the Hall mobility $\mu_{\mathrm{H}}$ for a n-type GaAs sample. 1) Temperature range where the scattering is characterized by a relaxation time (analysis range). 2) Temperature range where the scattering is not characterized by a relaxation time (optical phonons essentially).] 
- Lorsque la température croît, l'efficacité de la dispersion des électrons par les phonons optiques croît : ceci nécessiterait la prise en compte de ce mécanisme de diffusion pour le calcul de la mobilité. Or, la méthode développée ici utilise la description des processus de dispersion à partir du temps de relaxation : celle-ci ne peut être appliquée au cas des phonons optiques, l'énergie échangée lors de cette diffusion pouvant être grande par rapport à l'énergie incidente du porteur. Ehrenreich [13] en utilisant une technique variationnelle pour résoudre l'équation de Boltzmann, obtient l'équivalent d'un temps de relaxation pour la dispersion par les phonons optiques : cependant, cette solution ne convient pas pour GaAs lorsque $150 \mathrm{~K}<T<300 \mathrm{~K}$ et demeure très incertaine quand d'autres mécanismes de diffusion sont simultanément efficaces [14]. De ce fait, nous avons limité supérieurement la zone d'exploitation à des températures telles que cette dispersion par les phonons optiques puisse être légitimement négligée (cf. Fig. 2). Notons que le choix de la zone de température à analyser est guidé par les résultats des travaux de Stillman et al. [4] qui ont défini dans le cas de n-GaAs les limites du domaine où la dispersion par les impuretés ionisées est prépondérante. D'autre part, le domaine de température choisi

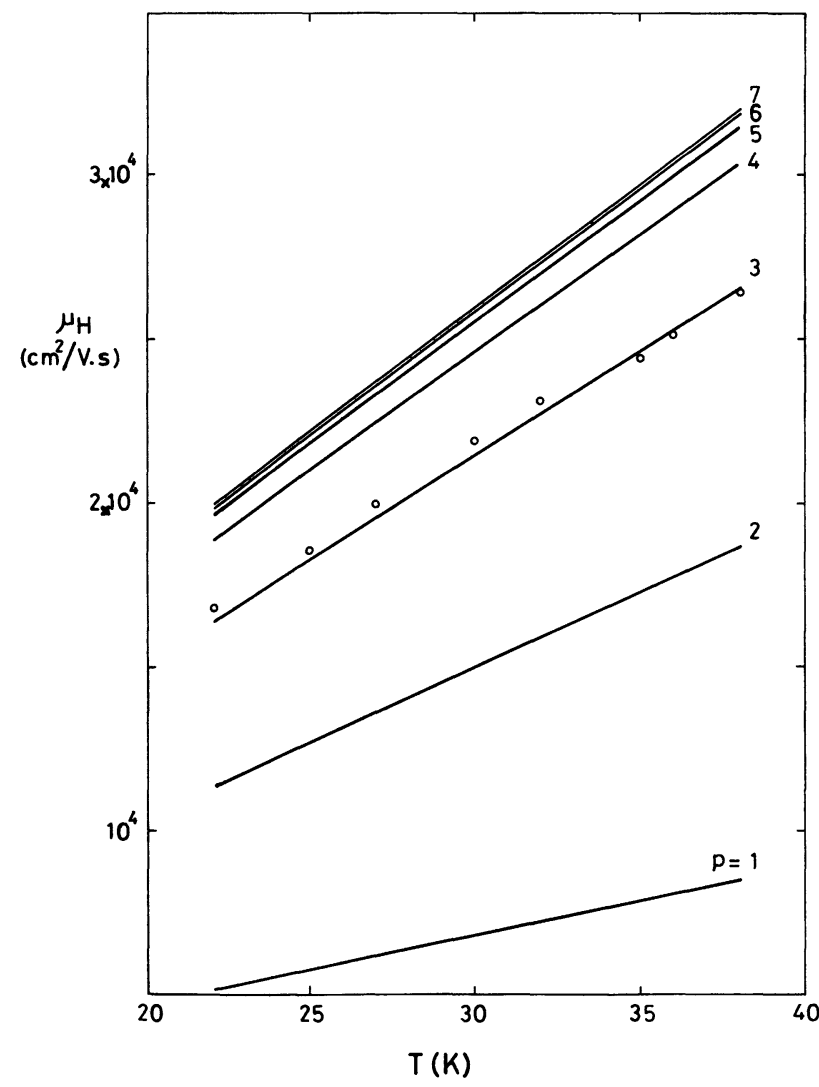

Fig. 3. - Résultats numériques obtenus à la fin de chaque cycle (ordre $p$ ) pour une boucle de calcul donnée $\left(r_{\mathrm{H}}(300 \mathrm{~K})\right.$ constant). (Les cercles représentent les points expérimentaux de l'échantillon correspondant).

[Numerical results obtained at the end of each cycle (order $p$ ) for one computation loop ( $r_{H}(300 \mathrm{~K})$ constant). (The circles represent the experimental points of the corresponding sample.)] peut être affiné compte tenu des résultats de calcul obtenus.

Des fonctions test ont été introduites dans le déroulement d'un cycle de calcul. Tout d'abord, les valeurs de $\varepsilon_{\mathbf{D}}$ et de $N_{\mathrm{A}}$ obtenues en fin de première partie sont celles qui minimisent la fonction erreur $f$ définie par :

$$
f=\sum_{i=1}^{i=M}\left[\frac{n^{(p, q)}\left(T_{\mathrm{i}}\right)-n^{(p, q) \mathrm{calc}}\left(T_{\mathrm{i}}\right)}{n^{(p, q)}\left(T_{\mathrm{i}}\right)}\right]^{2}
$$

où $n^{(p, q) \text { calc }}\left(T_{\mathfrak{i}}\right)$ est la concentration électronique calculée à partir de l'équation (10).

A la fin de la deuxième partie, la convergence des résultats est observée grâce à une fonction $g(p, q)$ obtenue à partir des mobilités de Hall calculées au cours du cycle précédent $\mu_{\mathrm{H}}^{(p-1, q) \text { calc }}\left(T_{\mathfrak{i}}\right)$ et celles obtenues à la fin du cycle $p$ et telle que :

$g(p, q)=\frac{1}{M} \sum_{i=1}^{i=M}\left|\frac{\mu_{\mathrm{H}}^{(p-1, q) \text { calc }}\left(T_{\mathrm{i}}\right)-\mu_{\mathrm{H}}^{(p, q) \text { calc }}\left(T_{\mathrm{i}}\right)}{\mu_{\mathrm{H}}^{(p, q) \text { calc }}\left(T_{\mathrm{i}}\right)}\right|$.

Si la valeur obtenue pour $g(p, q)$ est jugée trop importante (dans notre application supérieure à $10^{-2}$ ) un

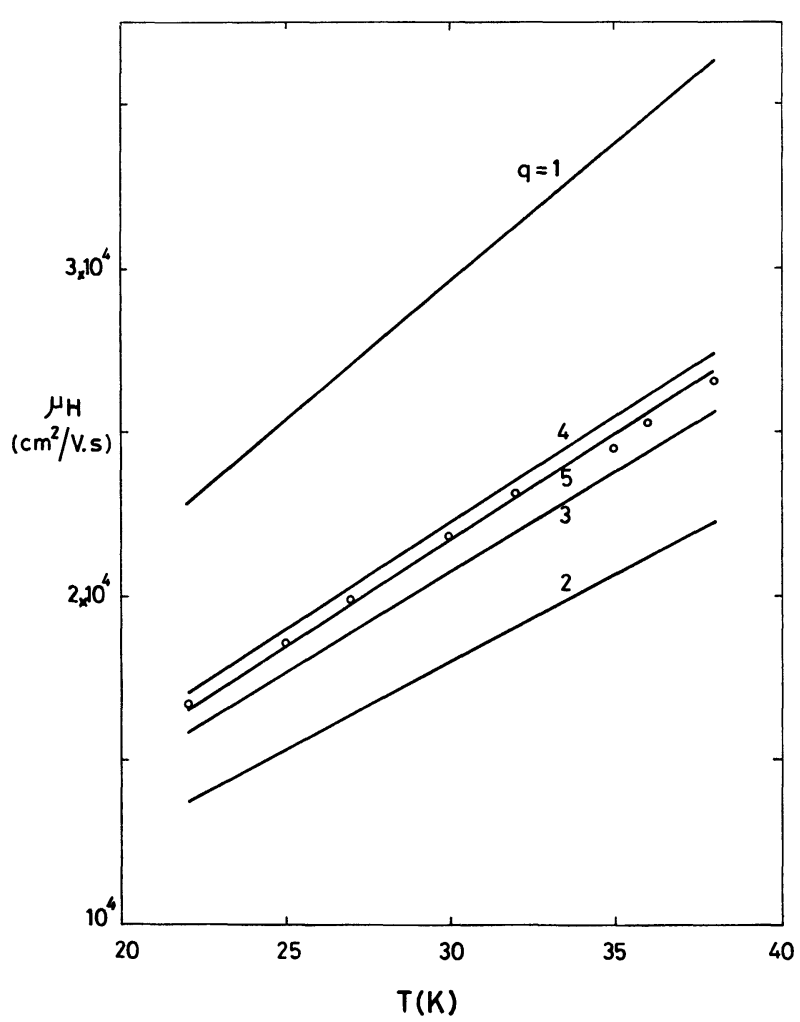

Fig. 4. - Résultats numériques obtenus à la fin de plusieurs boucles de calcul (ordre $q$ ) en fonction de la variation de $r_{\mathrm{H}}(300 \mathrm{~K})$. 1) $\left.r_{\mathrm{H}}(300 \mathrm{~K})=1,15.2\right) r_{\mathrm{H}}(300 \mathrm{~K})=1,30$. 3) $\left.\left.r_{\mathrm{H}}(300 \mathrm{~K})=1,25.4\right) r_{\mathrm{H}}(300 \mathrm{~K})=1,23.5\right) r_{\mathrm{H}}(300 \mathrm{~K})=1,238$. (les cercles représentent les points expérimentaux de l'échantillon correspondant).

[Numerical results obtained at the end of several loops of computation loops (order $q$ ) as a function of $r_{\mathrm{H}}(300 \mathrm{~K})$. 1) $\left.\left.r_{\mathrm{H}}(300 \mathrm{~K})=1.15 .2\right) r_{\mathrm{H}}(300 \mathrm{~K})=1.30 .3\right) r_{\mathrm{H}}(300 \mathrm{~K})=1.25$. 4) $r_{\mathrm{H}}(300 \mathrm{~K})=1.23$. 5) $r_{\mathrm{H}}(300 \mathrm{~K})=1.238$. (The circles represent the experimental points of the corresponding sample.)] 
autre cycle est entrepris partant de nouvelles valeurs de $r_{\mathrm{H}}^{(p, q)}\left(T_{\mathrm{i}}\right)$ déduites de l'expression (8).

Dans le cas contraire, un test $S(p, q)$ est introduit pour comparer les résultats obtenus expérimentalement avec ceux calculés

$$
S\left(p_{\mathrm{f}}, q\right)=\frac{1}{M} \sum_{i=1}^{i=M}\left[\frac{\mu_{\mathrm{H}}^{\exp }\left(T_{\mathrm{i}}\right)-\mu_{\mathrm{H}}^{\left(p_{\mathrm{f}}, q\right) \mathrm{calc}}\left(T_{\mathrm{i}}\right)}{\mu_{\mathrm{H}}^{\exp }\left(T_{\mathrm{i}}\right)}\right]^{2}
$$

où $p_{\mathrm{f}}$ représente le numéro du dernier cycle de la boucle d'ordre $q$ en cours de calcul.

Pour une valeur de $S\left(p_{\mathrm{f}}, q\right)$ qui n'est pas satisfaisante (supérieure à $10^{-4}$ pour notre application) une nouvelle boucle est effectuée en prenant une valeur différente de $r_{\mathrm{H}}^{(q)}(300 \mathrm{~K})$, ce paramètre étant ajusté jusqu'à l'obtention d'un bon accord entre les mesures expérimentales de mobilité de Hall et celles ainsi calculées.

La rapidité de la convergence des résultats peut être appréciée sur la figure 3 qui représente un exemple de variations des valeurs de $\mu_{\mathrm{H}}^{(p, q) \text { calc }}\left(T_{\mathrm{i}}\right)$ pour les 7 premiers cycles d'une boucle: les valeurs de $g(p, q)$ correspondant à cet exemple sont regroupées dans le tableau ci-dessous :

\section{TABLEAU I}

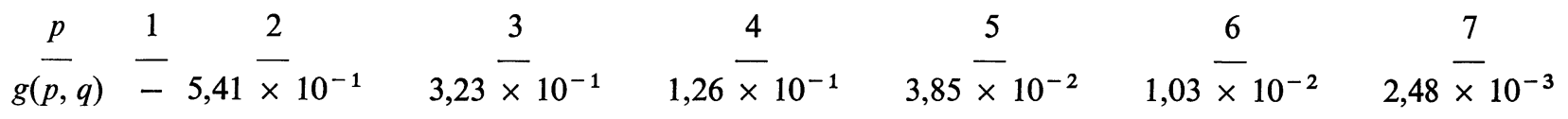

On remarque ainsi la décroissance de la fonction $g(p, q)$ après un nombre de cycles restreint, ce qui se traduit sur la figure 3 par un resserrement des courbes représentatives de $\mu_{\mathrm{H}}^{(p, q) \text { calc }}\left(T_{\mathrm{i}}\right)$ lorsque $p$ augmente. En pratique, l'étude de cette convergence montre qu'audelà de 6 cycles de calcul, la fonction $g(p, q)$ est toujours très inférieure à $10^{-2}$. La figure 3 , qui illustre les résultats d'une boucle de calcul intermédiaire, montre clairement que les valeurs calculées ne convergent pas vers les données expérimentales et la figure 4 représente l'évolution des résultats obtenus à la fin de chaque boucle en fonction du choix de $r_{\mathrm{H}}^{(q)}(300 \mathrm{~K})$ donc de celui de la différence $\left(N_{\mathrm{D}}-N_{\mathrm{A}}\right)$. Le terme $r_{\mathrm{H}}^{(q)}(300 \mathrm{~K})$ apparaît bien comme le seul paramètre ajustable de cette méthode. Le sens de la variation à donner à $r_{\mathrm{H}}(300 \mathrm{~K})$ est défini par le signe de l'expression suivante

$$
D\left(p_{\mathrm{f}}, q\right)=\frac{1}{M} \sum_{i=1}^{i=M}\left[\frac{\mu_{\mathrm{H}}^{\exp }\left(T_{\mathrm{i}}\right)-\mu_{\mathrm{H}}^{\left(p_{\mathrm{f}}, q\right) \text { calc }}\left(T_{\mathrm{i}}\right)}{\mu_{\mathrm{H}}^{\exp }\left(T_{\mathrm{i}}\right)}\right]
$$

La figure 4 montre un exemple d'évolution des valeurs de $\mu_{\mathrm{H}}^{\left(p_{\mathrm{f}}=6, q\right) \text { calc }}\left(T_{\mathrm{i}}\right)$ pour 5 boucles de 6 cycles de calcul, en fonction du choix du paramètre $r_{\mathrm{H}}^{(q)}(300 \mathrm{~K}):$ les variations correspondantes de la fonction $S\left(p_{\mathrm{p}}=6, q\right)$

\begin{tabular}{|c|c|c|c|c|c|}
\hline$q$ & 1 & 2 & 3 & 4 & 5 \\
\hline$S\left(p_{\mathrm{f}}=6, q\right)$ & $\overline{0,132}$ & $2,92 \times 10^{-2}$ & $2,14 \times 10^{-3}$ & $1,94 \times 10^{-4}$ & $1,35 \times 10^{-4}$ \\
\hline
\end{tabular}
sont précisées dans le tableau II ci-dessous :

TABLEAU II

Il convient de noter que le choix arbitraire de la première valeur de $r_{\mathrm{H}}(300 \mathrm{~K})$ entre 1,0 et $1,2 \mathrm{n}$ 'a, en pratique, aucune influence sur la valeur finale de ce paramètre ; cette première valeur de $r_{\mathrm{H}}(300 \mathrm{~K})$ influe simplement sur le nombre de boucles à réaliser pour arriver à un accord déterminé entre valeurs calculées et expérimentales de la mobilité de Hall.

Lorsque le calcul est arrêté, c'est-à-dire lorsque la valeur de la fonction $S\left(p_{\mathrm{f}}, q\right)$ devient inférieure à une valeur choisie, les résultats finals obtenus pour l'échantillon de n-GaAs analysé regroupent les concentrations $N_{\mathrm{D}}$ et $N_{\mathrm{A}}$ en éléments donneurs et accepteurs, l'énergie d'activation thermique $\varepsilon_{\mathrm{D}}$ du niveau donneur et, sur le domaine de température étudié, (compris entre $T_{1}$ et $T_{\mathrm{M}}$ ), les valeurs du facteur de dispersion $r_{\mathrm{H}}(T)$, de la mobilité de Hall $\mu_{\mathrm{H}}(T)$ et de la mobilité de conduction $\mu(T)$.

4. Conclusion. - La méthode que nous venons de décrire a été utilisée pour analyser les mesures expéri- mentales concernant des couches épitaxiées de n-GaAs et les résultats obtenus ont été publiés antérieurement [6]. Le programme de calcul a été traité sur l'ordinateur CII $10070 \mathrm{du}$ Centre Interuniversitaire de Calcul de Bretagne. Il faut remarquer que le terme $r_{\mathbf{H}}(300 \mathrm{~K})$ qui apparaît ici comme paramètre, prend des valeurs finales de l'ordre de 1,2 valeurs voisines de celles citées par d'autres auteurs [5] [8] et résultant d'approches différentes.

Nous avons essayé d'introduire dans cette méthode la description de la dispersion des électrons par les phonons optiques formulée par Ehrenreich [13] sous la forme d'un équivalent de temps de relaxation : les résultats obtenus pour la mobilité de Hall ont toujours présenté un écart important avec les valeurs expérimentales. Nous avons donc abandonné l'utilisation de cette description, limitant le domaine de température analysé à celui précisé au chapitre 3 pour que la diffusion par les phonons optiques puisse y être totalement négligée. 
La précision de cette méthode dépend essentiellement de la qualité des données expérimentales utilisées, puisque chaque étape de calcul peut être menée avec la précision désirée. Cependant, lorsque la concentration en éléments donneurs dépasse quelque $10^{16} \mathrm{~cm}^{-3}$, l'énergie d'activation thermique $\varepsilon_{D}$ tend vers 0 [15] et l'emploi de l'équation de neutralité (10) devient inadapté. Pour les échantillons de concentration en impuretés inférieure, cette méthode permet la prise en compte de la valeur réelle du facteur de dispersion $r_{\mathbf{H}}$ qui peut atteindre et dépasser 1,5 dans le cas d'échantillons de grande pureté et dans le domaine de température où est réalisée cette analyse. Pour la connaissance des densités $N_{\mathrm{D}}$ et $N_{\mathrm{A}}$ en impuretés, une précision supérieure est obtenue par rapport aux méthodes utilisées jusqu'alors et pour lesquelles le facteur de dispersion a été pris égal à 1 à toutes températures et pour tout échantillon.

\section{Bibliographie}

[1] Eddolss, D. V., Phys. Stat. Sol., 17 (1966) 67.

[2] Wolfe, C. M., Stillman, G. E. and Lindley, W. T., $J$. Appl. Phys. 41 (1970) 3088.

[3] Brooks, H., Adv. Elect. Elect. Phys. 7 (1955) 158.

[4] Wolfe, C. M., Stillman, G. E. and Dimmock, J. O., $J$. Appl. Phys. 41 (1970) 504.

[5] Stillman, G. E., Wolfe, C. M. and Dimmock, J. O., $J$. Phys. Chem. Solids 31 (1970) 1199.

[6] Lemoine, D., Pelletier, C., Rolland, S. and Granger, R. Phys. Stat. Sol (a) 34 (1976) 307.

[7] BeER, A. C., Galvanomagnetic effects in Semicond. (Acad. Press) 1963, chap. 5.
[8] Kravchenko, A. F., Kubalkova, S., Morozov, B. V., Polovinkin, V. G. and Skok, E. H., Phys. Stat. Sol. (b) 72 (1975) 221.

[9] Bardeen, J. and Shockley, W., Phys. Rev., 80 (1950) 72.

[10] Zook, J. D., Phys. Rev. A 136 (1964) 869.

[11] Erginsoy, C., Phys. Rev. 79 (1950) 1013.

[12] Luong, M. and Shaw, A. W., Phys. Rev. B 4 (1971) 2436.

[13] Ehrenreich, H., J. Appl. Phys. 32 (1961) 2155.

[14] Kuzel, R., Can. J. Phys. 47 (1969) 2261.

[15] Stillman, G. E., Wolfe, C. M. and Dimmock, J. O., GaAs and related compounds, (Institute of Physics London $\mathrm{n}^{\circ} 9$ 1970, 212. 